PROCEEDINGS OF THE

AMERICAN MATHEMATICAL SOCIETY

Volume 136, Number 7, July 2008, Pages 2343-2349

S 0002-9939(08)09213-7

Article electronically published on March 11, 2008

\title{
COMMENSURABILITY CLASSIFICATION OF A FAMILY OF RIGHT-ANGLED COXETER GROUPS
}

\author{
JOHN CRISP AND LUISA PAOLUZZI
}

(Communicated by Alexander N. Dranishnikov)

\begin{abstract}
We classify the members of an infinite family of right-angled Coxeter groups up to abstract commensurability.
\end{abstract}

\section{INTRODUCTION}

A number of papers have recently dealt with the problem of determining the automorphism groups and isomorphism classification of Coxeter groups, to the point where these questions appear to be more or less resolved at present. We refer the reader to the recent survey [4] which cites numerous contributions to the subject including, notably, the thesis of P.-E. Caprace 1] (see also [2, which deals mainly with Artin groups but contains a partial result on Coxeter groups not cited elsewhere). The classification of Coxeter groups up to abstract commensurability, on the other hand, appears to be an interesting and relatively unexplored problem. Two groups $G$ and $H$ are said to be abstractly commensurable, written $G \sim H$, if they contain isomorphic finite index subgroups. We note that while the isomorphism rigidity of Coxeter groups is governed largely by the finite subgroup structure, these features are not detected by abstract commensurators. Indeed, every Coxeter group is linear and hence virtually torsion free. The abstract commensurability class of a Coxeter group therefore reflects more the large scale geometry of the group than does the isomorphism class.

To each simplicial graph $\Gamma$ one associates the right-angled Coxeter group $W(\Gamma)$ given by the presentation

$$
\left.W(\Gamma)=\langle\operatorname{vert}(\Gamma)| u v=v u \text { if }\{u, v\} \in \operatorname{edge}(\Gamma), u^{2}=1 \text { for all } u \in \operatorname{vert}(\Gamma)\right\rangle .
$$

In this paper we consider the classification up to abstract commensurability of the following rather special family of right-angled Coxeter groups, suggested to the authors by B. Mühlherr. For $m, n \geq 1$, we define the group

$$
W_{m, n}=W\left(\Gamma_{m, n}\right)
$$

where $\Gamma_{m, n}$ denotes the graph which consists of a circuit of length $m+4$ and a circuit of length $n+4$ which are identified along a common subpath of edge-length 2 as shown in Figure[1(i). By a result of Radcliffe [5], two right-angled Artin groups are isomorphic if and only if their defining graphs are isomorphic. Groups $W_{m, n}$

Received by the editors October 12, 2006, and, in revised form, April 20, 2007.

2000 Mathematics Subject Classification. Primary $20 \mathrm{~F} 36$.

Key words and phrases. Abstract commensurability, right-angled, Coxeter group.

(C)2008 American Mathematical Society 


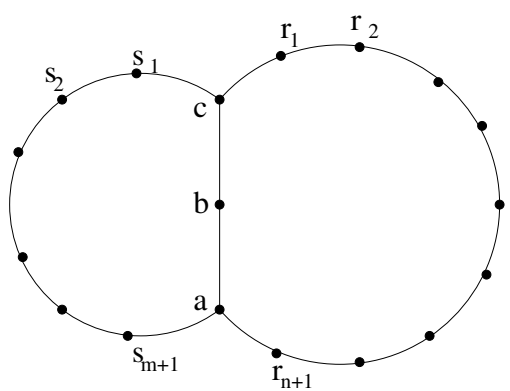

(i)

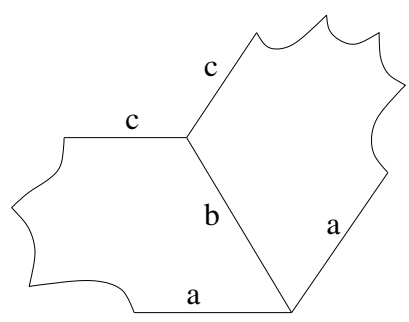

(ii)

Figure 1. (i) The graph $\Gamma_{m, n}$ and (ii) the orbifold $\mathcal{O}_{m, n}$

and $W_{k, l}$ are therefore isomorphic if and only if $\{m, n\}=\{k, l\}$. In this paper we show that the rational number $\frac{m}{n} \in(0,1]$ is a complete commensurability invariant within our family of groups $W_{m, n}$.

Theorem 1.1. Let $1 \leq m \leq n$ and $1 \leq k \leq l$. Then $W_{m, n}$ and $W_{k, l}$ are abstractly commensurable if and only if $\frac{m}{n}=\frac{k}{l}$.

\section{The GRoup $W_{m, n}$ AND ITS Finite INDEX SUBGROUPS}

2.1. The group $W_{m, n}$. If $C_{N}$ denotes the graph which is a circuit of length $N$, then, for $N \geq 5, W\left(C_{N}\right)$ is isomorphic to a co-compact subgroup of $\operatorname{Isom}\left(\mathbb{H}^{2}\right)$ generated by the reflections in the sides of a right-angled hyperbolic $N$-gon $\mathcal{P}_{N}$, which we view here as a reflection orbifold. In particular $W\left(C_{N}\right)$ is virtually a closed hyperbolic surface group. Note that the groups $W\left(C_{N}\right)$ are all commensurable for $N \geq 5$, while on the other hand, $W\left(C_{N}\right)$ is virtually abelian, when $N=4$, and finite when $N=3$. The reflections in any three consecutive faces $a, b, c$ of $\mathcal{P}_{N}$ will generate a subgroup $D_{\infty} \times \mathbb{Z} / 2 \mathbb{Z}$ where $D_{\infty}$ denotes the infinite dihedral group (generated by $a$ and $c$ ). The right-angled Coxeter group $W_{m, n}$ may therefore be viewed as an amalgamated product

$$
W_{m, n}=W\left(C_{m+4}\right) \star_{D_{\infty} \times \mathbb{Z} / 2 \mathbb{Z}} W\left(C_{n+4}\right) .
$$

This group may also be described as the fundamental group of the reflection orbifold $\mathcal{O}_{m, n}$ obtained by gluing a copy of $\mathcal{P}_{m+4}$ and a copy of $\mathcal{P}_{n+4}$ together along a common reflection edge (for the generator $b$ ). See Figure 1(ii).

2.2. Hyperbolic structure. Note that, for each $N \geq 5$, there are infinitely many (in fact a continuous family of) non-conjugate co-compact subgroups of $\operatorname{Isom}\left(\mathbb{H}^{2}\right)$ which are isomorphic to the group $W\left(C_{N}\right)$. This is because there is a continuous family of pairwise non-isometric, right-angled $N$-gons in the hyperbolic plane, for each $N \geq 5$. Note also that for any $N \geq 5$ it is possible to find right-angled $N$ gons with arbitrarily small shortest side-length. For this reason, one can always choose right-angled orbifolds $\mathcal{P}_{m+4}$ and $\mathcal{P}_{n+4}$ of constant curvature -1 so as to have at least one side-length in common. Gluing by an isometry of the common edge then produces a piecewise hyperbolic version of the orbifold $\mathcal{O}_{m, n}$ with constant curvature -1 . This choice of metric on $\mathcal{O}_{m, n}$ also induces a piecewise hyperbolic 
metric on any of its orbifold covering spaces with the nice property that every planar convex simply connected subspace is isometric to a convex subspace of $\mathbb{H}^{2}$. Moreover it is easily seen that these structures are all locally $\mathrm{CAT}(-1)$, and this gives a straightforward proof that the group $W\left(C_{N}\right)$ is a hyperbolic group in the sense of Gromov.

2.3. Tiled surfaces. We now make some purely topological observations. Let $m \geq$ 1 , and define the $m$-tile $P_{m+4}$ to be the underlying polygon of $\mathcal{P}_{m+4}$ equipped with an orientation of each edge and a labelling which distinguishes the $m+4$ edges (or sides). By an $m$-type surface we mean a compact surface (with or without boundary, and not necessarily connected) tiled with $m$-tiles in such a way that (i) tiles are glued only along edges with the same label and respecting the orientation; and (ii) each interior vertex has valence 4 and each vertex on the boundary has valence 2 . Note that (i) and (ii) imply that every edge on a given boundary component of the surface carries the same label.

We note that an $m$-type surface may always be constructed as the underlying tiled surface associated with an orbifold covering of $\mathcal{P}_{m+4}$. The only added constraints are that each boundary component should be a geodesic reflector curve.

Lemma 2.1. If $S$ is any compact $m$-type surface, with or without boundary, then it has Euler characteristic $\chi(S)=\frac{-m|S|}{4}$ where $|S|$ denotes the number of $m$-tiles in the tiling of $S$.

Proof. For a closed $m$-type surface, this is a straightforward calculation: one has $|S|$ faces, $\frac{(m+4)|S|}{2}$ edges, and $\frac{(m+4)|S|}{4}$ vertices in the polygonal tiling of $S$. The case of a surface with boundary can be reduced to the closed case by doubling along the boundary (this operation simultaneously doubles both the Euler characteristic of $S$ and the value $\frac{-m|S|}{4}$ ).

2.4. Finite index subgroups. Any finite index subgroup of $W_{m, n}$ may be described as the (orbifold) fundamental group of a finite sheeted orbifold covering of $\mathcal{O}_{m, n}$. When the finite index subgroup $G<W_{m, n}$ is torsion free, then it is the genuine fundamental group, $G=\pi_{1}\left(X_{m, n}\right)$, of a connected 2-complex $X_{m, n}$, which is an orbifold covering of $\mathcal{O}_{m, n}$ with the following structure:

(i) $X_{m, n}$ is the union of a pair of not necessarily connected closed surfaces $\Sigma_{1}$ and $\Sigma_{2}$ (each embedded in $X_{m, n}$ ) which intersect transversely in a collection $\mathcal{C}$ of mutually disjoint simple closed curves: $X_{m, n}=\Sigma_{1} \cup_{\mathcal{C}} \Sigma_{2}$.

(ii) The surface $\Sigma_{1}$ (resp. $\Sigma_{2}$ ) has the structure of an $m$-type surface (resp. $n$-type surface).

(iii) There is a distinguished label $b$ common to $\Sigma_{1}$ and $\Sigma_{2}$ (corresponding to the generator $b$ as shown in Figure 1(i)) such that each $b$-edge in $\Sigma_{1}$ is identified with a unique $b$-edge in $\Sigma_{2}$, and $\Sigma_{1} \cap \Sigma_{2}$ (which is just the union of the curves in $\mathcal{C}$ ) is exactly equal to the union of all $b$-edges.

Note that it is easily seen (by construction) that $W_{m, n}$ is virtually torsion free.

\section{Proof of Theorem 1.1}

3.1. Commensurable groups. We first observe that if $\frac{m}{n}=\frac{k}{l}$ (for $m, n, k, l \geq 1$ ), then the groups $W_{m, n}$ and $W_{k, l}$ are abstractly commensurable. For any positive integer $R$ we can construct a degree $R$ orbifold covering $R \mathcal{O}_{m, n} \rightarrow \mathcal{O}_{m, n}$, by unfolding $R$ times along reflectors of type $a$ and $c$, in such a way that the edges labelled $b$ will 


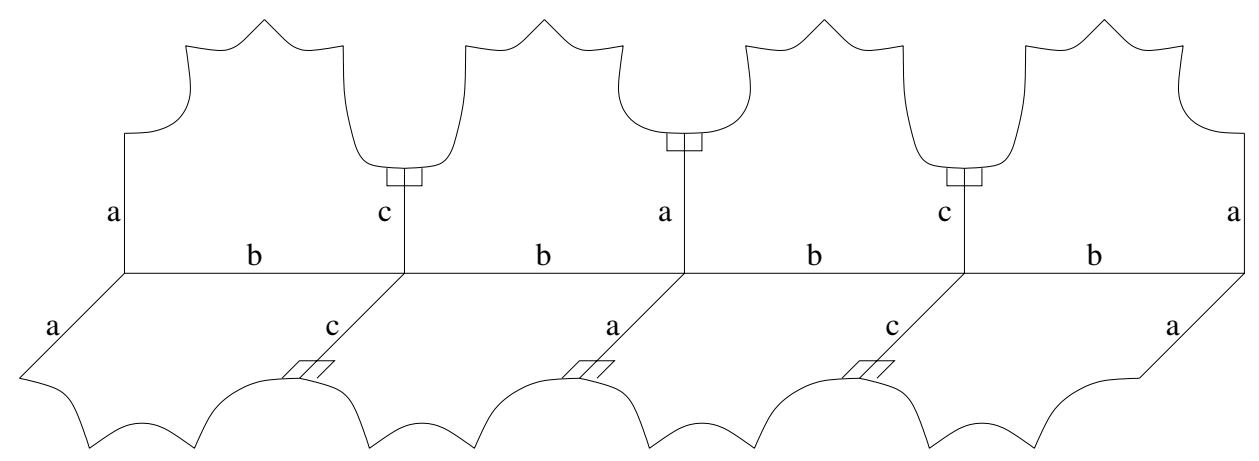

Figure 2. The orbifold $R \mathcal{O}_{m, n}$

form a geodesic path of length $R$. The resulting orbifold structure is illustrated in Figure 2. An easy counting argument shows that $R \mathcal{O}_{m, n}=\mathcal{O}_{R m, R n}$ as topological orbifolds. This shows that $W_{R m, R n}$ is a finite index subgroup of $W_{m, n}$ for all $R$. In particular, if $\frac{m}{n}=\frac{k}{l}$, then $W_{k m, k n}=W_{m k, m l}$ is a finite index subgroup of both $W_{m, n}$ and $W_{k, l}$, proving commensurability.

3.2. Rigidity of the complex $X_{m, n}$. Throughout this section we use the notation $X_{m, n}$ to refer to an orbifold covering of $\mathcal{O}_{m, n}$ which corresponds to some torsion free finite index subgroup of $W_{m, n}$, for $m, n \geq 1$, as described in Subsection 2.4.

Proposition 3.1. Let $X=X_{m, n}$ (resp. $X^{\prime}=X_{k, l}$ ) denote an orbifold cover of $\mathcal{O}_{m, n}$ (resp. $\mathcal{O}_{k, l}$ ) corresponding to a torsion free finite index subgroup of $W_{m, n}$ (resp. $\left.W_{k, l}\right)$. Then any isomorphism between $\pi_{1}(X)$ and $\pi_{1}\left(X^{\prime}\right)$ is induced by a homeomorphism of the two complexes $X$ and $X^{\prime}$.

Proposition 3.1 is a special case of a theorem of J.-F. Lafont [3, Theorem 1.2]. Indeed, the complexes $X_{m, n}$ corresponding to torsion free finite index subgroups of $W_{m, n}$ are basic examples of simple, thick, 2-dimensional hyperbolic P-manifolds (see [3, Definition 2.3]). For this reason we shall only give here a sketch of the proof of Proposition 3.1. Our approach differs slightly from that of Lafont, and so may be of independent interest.

We first recall that the complexes $X$ and $X^{\prime}$ are negatively curved with Gromov hyperbolic fundamental groups. Any isomorphism from $\pi_{1}(X)$ to $\pi_{1}\left(X^{\prime}\right)$ therefore induces a homeomorphism between the boundaries of the universal coverings $\widetilde{X}$ and $\widetilde{X}^{\prime}$ of the two complexes. The strategy is to use this homeomorphism between the boundaries to construct a bi-equivariant homeomorphism between $\widetilde{X}$ and $\widetilde{X}^{\prime}$. This clearly suffices to establish a homeomorphism from $X$ to $X^{\prime}$ which induces the given isomorphism of fundamental groups.

Note that the universal covering complex $\widetilde{X}$ may be viewed as a collection of hyperbolic planes glued together along certain geodesic lines, which we shall call double geodesics. The closures of the components obtained by removing from $\widetilde{X}$ all double geodesics are convex planar regions of the hyperbolic plane. Note also that, by pasting together planar regions along double geodesics, one can obtain new hyperbolic planes embedded in $\widetilde{X}$.

The first step of the proof is to show that every circle $S$ embedded in $\partial \widetilde{X}$ is the boundary of a unique hyperbolic plane embedded in $\widetilde{X}$. This plane can be 
characterised as the set of all points $p \in \widetilde{X}$ such that $S$ is homotopically non-trivial in $\widetilde{X} \cup \partial \widetilde{X}$. We leave the details of this step to the reader.

As a consequence, one can readily show that points $x, y$ in the boundary of $\widetilde{X}$ are the endpoints of a double geodesic if and only if there are circles $S_{1}, S_{2}$ in the boundary with $S_{1} \cap S_{2}=\{x, y\}$. Since a similar statement holds for $\widetilde{X}^{\prime}$, this establishes a bijection $F$ from the double geodesics of $\tilde{X}$ to those of $\widetilde{X}^{\prime}$. Now observe that one can define an adjacency relation on the set of double geodesics of $\widetilde{X}$ by saying that two double geodesics are adjacent if they are not separated by a third double geodesic. Equivalently, two double geodesics are adjacent if and only if they are not separated in the boundary of $\widetilde{X}$ by another double geodesic. This adjacency relation is necessarily preserved under the bijection $F$. Moreover, it is straightforward to see that a set of mutually adjacent double geodesics is maximal if and only if it coincides with the set of all double geodesics which lie in the closure of a single planar region. Thus the bijection $F$ induces a bijection $L$ from the set of planar regions of $\widetilde{X}$ to those of $\widetilde{X}^{\prime}$.

We now need to exhibit a suitable homeomorphism from each planar region of $\widetilde{X}$ to its image under $L$. The idea is as follows: fix a planar region $R$ in $\widetilde{X}$ and consider its quotient by the action of its stabiliser in $\pi_{1}(X)$; this is a compact subsurface of $X$ with totally geodesic boundary. Choose a family of properly embedded, pairwise disjoint arcs on the surface which cut it into a polygon. Note that, since the boundary is geodesic, these arcs can be chosen to be geodesic and orthogonal to the boundary. This polygonal decomposition lifts to an equivariant tessellation of the planar region by means of an infinite family of pairwise disjoint geodesic segments orthogonal to the double geodesics on the boundary. Using the fact that two ultraparallel geodesics admit a unique perpendicular, the given bijection among double geodesics allows us to construct a family of geodesic segments perpendicular to the boundary in the planar region $R^{\prime}=L(R)$ of $\widetilde{X}^{\prime}$. We claim that the new segments are mutually disjoint and therefore define a tessellation of $R^{\prime}$.

To see the claim, note that the set $D(R)$ of double geodesics in the boundary of a planar region $R$ has a natural cyclic ordering. In fact, this ordering depends only on the topology of the boundary of $\widetilde{X}$ since it can be recovered from the cyclic ordering of points in any circle in the boundary of $\widetilde{X}$ which passes through the endpoints of all geodesics in $D(R)$. (By our previous remark, every such circle is exactly the boundary of a complete hyperbolic plane containing $R$.) This cyclic ordering is therefore preserved under the bijection $F$ and is sufficient to determine whether or not the common perpendiculars to two pairs of double geodesics intersect.

We now have a combinatorial mapping from a tessellation of $R$ to one of $R^{\prime}$ which, by construction, is bi-equivariant with respect to the stabilisers of $R$ and $R^{\prime}$ respectively. Clearly this may be extended to a genuine equivariant homeomorphism from $R$ to $R^{\prime}$.

To finish, note that we may repeat the above construction for every region in a manner which is equivariant with respect to the full group action. Finally, the definition of these "local" homeomorphisms may be adjusted in an equivariant way along the double geodesics so that they can be glued together to give a global bi-equivariant homeomorphism from $\widetilde{X}$ to $\widetilde{X}^{\prime}$. 
3.3. Completing the proof of Theorem 1.1. We are now in a position to prove that if $W_{m, n}$ (with $m \leq n$ ) and $W_{k, l}$ (with $k \leq l$ ) are abstractly commensurable groups, then $\frac{m}{n}=\frac{k}{l}$.

Since $W_{m, n}$ is virtually torsion free we may suppose that $W_{m, n}$ and $W_{k, l}$ contain isomorphic torsion free finite index subgroups. In other words there exist complexes $X_{m, n}$ and $X_{k, l}$ (as above) such that $\pi_{1}\left(X_{m, n}\right) \cong \pi_{1}\left(X_{k, l}\right)$. By Proposition 3.1 we have a homeomorphism between these two complexes $X_{m, n}$ and $X_{k, l}$.

Write $X_{m, n}=\Sigma_{1} \cup_{\mathcal{C}} \Sigma_{2}$ and $X_{k, l}=\Sigma_{1}^{\prime} \cup_{\mathcal{C}^{\prime}} \Sigma_{2}^{\prime}$, where $\Sigma_{1}$ is an $m$-type surface, $\Sigma_{2}$ an $n$-type surface, $\Sigma_{1}^{\prime}$ a $k$-type surface, and $\Sigma_{2}^{\prime}$ an $l$-type surface. Let $h: X_{m, n} \rightarrow$ $X_{k, l}$ denote the known homeomorphism. We observe that $h$ maps the collection of curves $\mathcal{C}$ bijectively onto the collection $\mathcal{C}^{\prime}$. By a singular edge of $X_{m, n}$ (respectively $X_{k, l}$ ) we simply mean an edge of the tiling which lies in $\mathcal{C}$ (resp. $\mathcal{C}^{\prime}$ ).

Define the following closed subcomplexes of $X_{m, n}$ :

$$
U=h^{-1}\left(\Sigma_{1}^{\prime}\right) \cap \Sigma_{1} \quad \text { and } \quad V=h^{-1}\left(\Sigma_{2}^{\prime}\right) \cap \Sigma_{2} .
$$

These are $m$-type and $n$-type surfaces respectively. Note that, swapping the roles of $\Sigma_{1}^{\prime}$ and $\Sigma_{2}^{\prime}$ if necessary, we may always suppose that the surface $U$ is non-empty.

We claim that the number $|U|$ of $m$-tiles in $U$ is equal to the number $|V|$ of $n$-tiles in $V$. Let $e$ denote any singular edge of $X_{m, n}$. Then $e$ belongs to exactly four tiles, two from each of $\Sigma_{1}$ and $\Sigma_{2}$. Let $R_{1}, Q_{1}$ denote those from $\Sigma_{1}$, and $R_{2}, Q_{2}$ those from $\Sigma_{2}$. Under the homeomorphism $h$, the edge $e$ is mapped into a singular curve in $\mathcal{C}^{\prime}$ and two of the four tiles $R_{1}, Q_{1}, R_{2}, Q_{2}$ will map into $\Sigma_{1}^{\prime}$ with the other two mapping into $\Sigma_{2}^{\prime}$. Clearly the number of tiles from $\left\{R_{1}, Q_{1}\right\}$ which map into $\Sigma_{1}^{\prime}$ is equal to the number of tiles from $\left\{R_{2}, Q_{2}\right\}$ which map into $\Sigma_{2}^{\prime}$. In other words, the number of tiles adjacent to $e$ which lie in $U$ is equal to the number which lie in $V$. Summing over all edges in $X_{m, n}$ and using the fact that each tile is adjacent to exactly one singular edge, we find that $|U|=|V|$. Note that since $U$ is non-empty this number is non-zero.

Now let $U^{\prime}=h(U)$ and $V^{\prime}=h(V)$. By a completely similar argument (with respect to the inverse map $h^{-1}$ rather than $h$ ) we conclude that $\left|U^{\prime}\right|=\left|V^{\prime}\right| \neq 0$, where here we are counting tiles in the complex $X_{k, l}$. Using Lemma 2.1 we now have

$$
\frac{m}{n}=\frac{m|U|}{n|V|}=\frac{\chi(U)}{\chi(V)}=\frac{\chi\left(U^{\prime}\right)}{\chi\left(V^{\prime}\right)}=\frac{k\left|U^{\prime}\right|}{l\left|V^{\prime}\right|}=\frac{k}{l} .
$$

This completes the proof of Theorem 1.1

\section{Closing Remarks}

While, in this paper, we consider only a very modest family of Coxeter groups, we feel that the treatment of this special case gives a good idea of the flavour of the more general problem, at least in the case of right-angled Coxeter groups of virtual dimension 2. We hope that a number of the ideas and arguments presented here will generalise to much larger classes of Coxeter groups.

\section{ACKNOWLEDGEMENTS}

We wish to thank François Gautero and Bernhard Mühlherr for valuable discussions. 


\section{REFERENCES}

1. P.-E. Caprace, "Abstract" homomorphisms of split Kac-Moody groups, Ph.D. thesis, Université Libre de Bruxelles, December 2005.

2. J. Crisp, Automorphisms and abstract commensurators of 2-dimensional Artin groups, Geometry and Topology 9 (2005) 1381-1441. MR2174269 (2006g:20057)

3. J.-F. Lafont, Diagram rigidity for geometric amalgamations of free groups, J. Pure Appl. Algebra 209 (2007) 771-780. MR2298855

4. B. Mühlherr, The isomorphism problem for Coxeter groups, in "The Coxeter Legacy", 1-15, Amer. Math. Soc., Providence, RI, 2006. MR2209018 (2006k:20084)

5. D. Radcliffe, Rigidity of graph products of groups, Algebr. Geom. Topol. 3 (2003) 1079-1088. MR2012965 (2004h:20056)

I.M.B. (UMR 5584 du CNRS), Université de Bourgogne, B.P. 47 870, 21078 Dijon, FRANCE

E-mail address: jcrisp@u-bourgogne.fr

I.M.B. (UMR 5584 Du CNRS), Université de Bourgogne, B.P. 47 870, 21078 Dijon, FRANCE

E-mail address: paoluzzi@u-bourgogne.fr 